\title{
INTERPRETATION OF RADIO OBSERVATIONS OF FH SERPENTIS
}

\author{
M. Friedjung \\ Institut d'Astrophysique \\ Paris, France
}

Seaquist and Palimaka (1977) interpreted the radio observations of this nova according to a "Hubble flow" model, in which all gas was efected at the same time, and for which the velocity of any point in the shell at a given time is proportional to its radial distance. Their model involving a thick shell contradicts observations made in other parts of the spectrum of this and other novae, so in the present study other possibilities have been examined.

It is assumed that most mass is in a thin shell ejected around maximum (most of the less massive continuously ejected gas having already collided with the outer shell), but which is inhomogeneous with differing optical thickness along different radil. In addition the electron temperature is supposed constant, while it is assumed that as the shell expands the law giving the ratio of the total areas subtended by inhomogeneitles of two different fixed optical thicknesses is unchanged. One then finds that the observations suggest that the Inhomogeneities expand in the radial direction, so that their radial extension is proportional to the time since ejection. Calculations to find the law of total areas are not yet completed, though laws where total area is proportional to a power of the optical thickness may be better than exponential laws.

\section{REFERENCE}

Seaquist, E.R., and Palimaka, J. 1977, Ap.J., 217, 781. 